\title{
Drag-shield drop tower residual acceleration optimisation
}

\author{
A Figueroa, F Sorribes-Palmer, M Fernandez De Pierola and \\ J Duran
}

Institute for Microgravity 'Ignacio Da Riva', Technical University of Madrid (UPM), Spain

E-mail: felix.sorribes@upm.es

Received 21 December 2015, revised 29 February 2016

Accepted for publication 29 April 2016

Published 19 May 2016

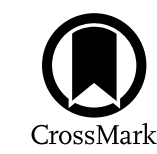

\begin{abstract}
Among the forces that appear in drop towers for microgravity experiments, aerodynamic drag plays a crucial role in the residual acceleration. Buoyancy can also be critical, especially at the first instances of the drop when the low speed of the experimental platform makes the aerodynamic drag small compared with buoyancy. In this paper the perturbation method is used to formulate an analytical model which has been validated experimentally. The experimental test was conduced by undergraduate students of aerospace engineering at the Institute of Microgravity 'Ignacio Da Riva' of the Technical University of Madrid (IDR/UPM) microgravity tower. The test helped students to understand the influence of the buoyancy on the residual acceleration of the experiment platform. The objective of the students was to understand the physical process during the drop, identify the main parameters involved in the residual acceleration and determine the most suitable configuration for the next drop tower proposed to be built at UPM.
\end{abstract}

Keywords: microgravity, drop tower, aerodynamics, buoyancy, drag-shield, educational

\section{Introduction}

Weightlessness is the environment attained when gravity is the only force acting on a body. In practice, other forces accelerate the system; this acceleration is defined as residual acceleration. In these conditions, a microgravity environment is achieved and the gravitational force does not dominate the physical phenomena that take place on the system (Malméjac et al 1981).

Experimentation in microgravity conditions is a useful tool in the development of space systems due to the differences in the behaviour of physical phenomena (Ruyters and 
Friederich 2006). For example, any system which involves the movement or management of fluids needs a thorough study prior to its use, because the change of conditions will carry big differences in the performance of those systems and in the behaviour of the fluids that are being used (Urban et al 2004, Li et al 2011).

In order to perform microgravity experiments, it is important to generate controlled microgravity environments. There are multiple ways of producing microgravity conditions: orbital platforms, sounding rockets, sounding balloons, parabolic flights, drop towers, etc (Thomas et al 2000, Reibaldi et al 2004).

Drop towers are structures designed for the performance of experiments under microgravity conditions by letting a body fall freely through them. Bodies fall freely through a tube, with the least possible interaction of external forces other than the gravitational pull, and forcing a fall acceleration as close as possible to the gravitational acceleration. In this manner, the apparent weight of the payload that is falling will be close to zero, hence being in a state of 'weightlessness' or 'microgravity' in which the effects of the external forces on the falling body are negligible (Kufner et al 2011).

In drop towers shorter than $30 \mathrm{~m}$, the most employed strategy to reduce the aerodynamic drag consists of covering the experimental platform with a drag-shield (Kaldeich 1995, Steinberg 2008, Osborne and Welch 2012). The drop tower located at the University Institute of Microgravity 'Ignacio Da Riva' of the Technical University of Madrid (IDR/UPM) belongs to this group. It was built using a decommissioned ventilation chimney spanning from the top flat roof of the building to the lower floor. It is a small drop tower that provides moderate $g$-levels during short periods of time at a reduced cost. It has been used mainly for academic research on the behaviour of liquid in microgravity environments (i.e. capillarydriven phenomena, Meseguer et al 2014).

A study of the experimental platform movement has been conducted, in order to understand the mechanisms that influence the microgravity level achieved. Two mathematical models have been developed to analyse the movement characteristics. One of the models includes the effect of the pressure gradient generated by the drag-shield acceleration, apart from the aerodynamic drag influence.

The accomplishment of this study is based on the knowledge acquired in the IDR/UPM drop tower. The objective of this study is to improve its configuration so as to carry out better quality experiments and design a reliable structure for the drag-shield. In these facilities an experiment has been performed to validate the theoretical models with the real movement of the platform. The proposed approach could be used to study possible enhancements of drop tower performance.

\section{Mathematical models}

As first approach, the gravity and the aerodynamic drag have been considered as the only forces that act on the drag-shield during its drop. According to this, the acceleration of the drag-shield can be calculated as

$$
\frac{\mathrm{d} v}{\mathrm{~d} t}=g-\frac{\rho S C_{\mathrm{D}}}{2 m} v^{2}
$$

where $g$ is the gravitational acceleration, $v$ is the drag-shield velocity, $m$ is the drag-shield mass, $\rho$ is the air density, $S$ the frontal drag-shield area and $C_{\mathrm{D}}$ the drag coefficient (only vertical velocity and acceleration are considered for the air drag effect). 

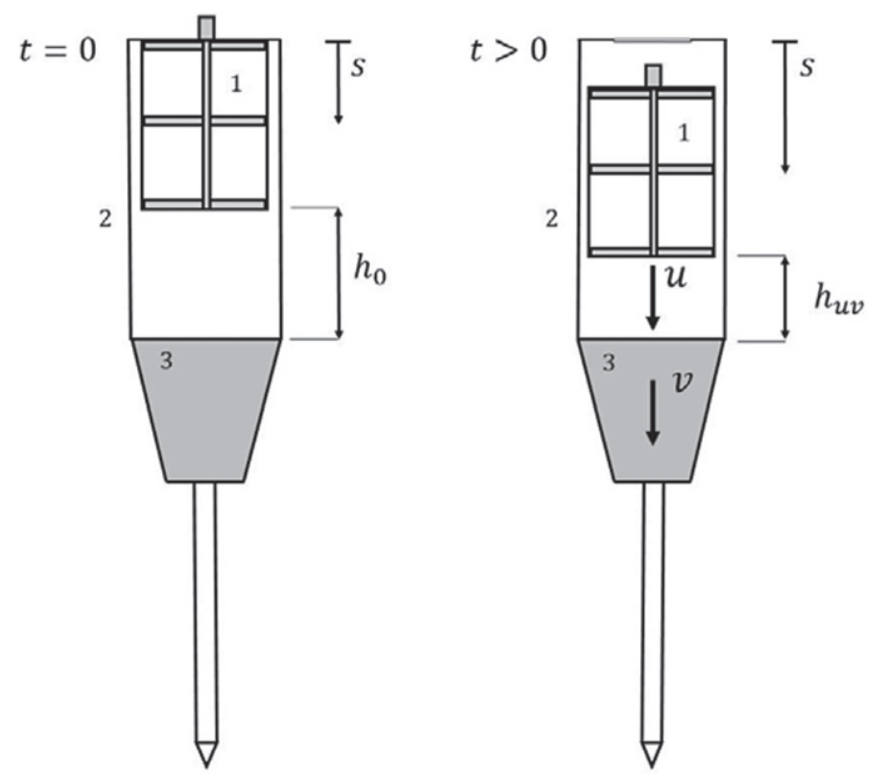

Figure 1. Sketch of the relative position of the experiment platform (1) and the dragshield (2) at the release instant, $t=0$, and during the fall, after release, $t>0$, (3) represents the foam housed at the bottom of the drag-shield to damp the impact between the experiment platform and the drag-shield.

When the experimental platform falls inside the drag-shield, as shown in figure 1 , the relative velocity between the air and the experimental platform is small, and therefore, the aerodynamic drag force is reduced. To model this force the velocity of the air contained in the drag-shield has been assumed equal to the drag-shield velocity. According to this assumption, the drag force which acts on the experimental platform is

$$
D_{\mathrm{p}}=\frac{1}{2} \rho S_{\mathrm{p}} C_{\mathrm{Dp}}(u-v)^{2},
$$

where $u$ is the platform velocity, $S_{\mathrm{p}}$ is the frontal experimental platform area and $C_{\mathrm{Dp}}$ is its drag coefficient. On the other hand, as it is assumed that the air moves with the same velocity as the drag-shield, its accelerations is also equal to the drag-shield one. By applying the equations of conservation of momentum to a finite volume of air, it is obtained

$$
\rho \frac{\mathrm{d} v}{\mathrm{~d} t}=-\frac{\mathrm{d} p}{\mathrm{~d} s},
$$

where $p$ is the air pressure and $s$ is the displacement of the platform with respect to the top of the drag-shield, with the same direction of the drop, as it is shown in figure 1. The pressure difference between the top and the bottom platform surfaces can be obtained by integration of equations (1) and (3). This way, the mentioned pressure difference is

$$
\Delta p=\rho l\left(g-\frac{\rho S C_{\mathrm{D}}}{2 m} v^{2}\right)
$$

where $l$ is the experimental platform length. The resultant force, $F_{\mathrm{p}}$, can be calculated by multiplying the pressure difference by the frontal area of the platform $S_{\mathrm{p}}$. Due to the nature of the mentioned force, it has been called buoyancy force 


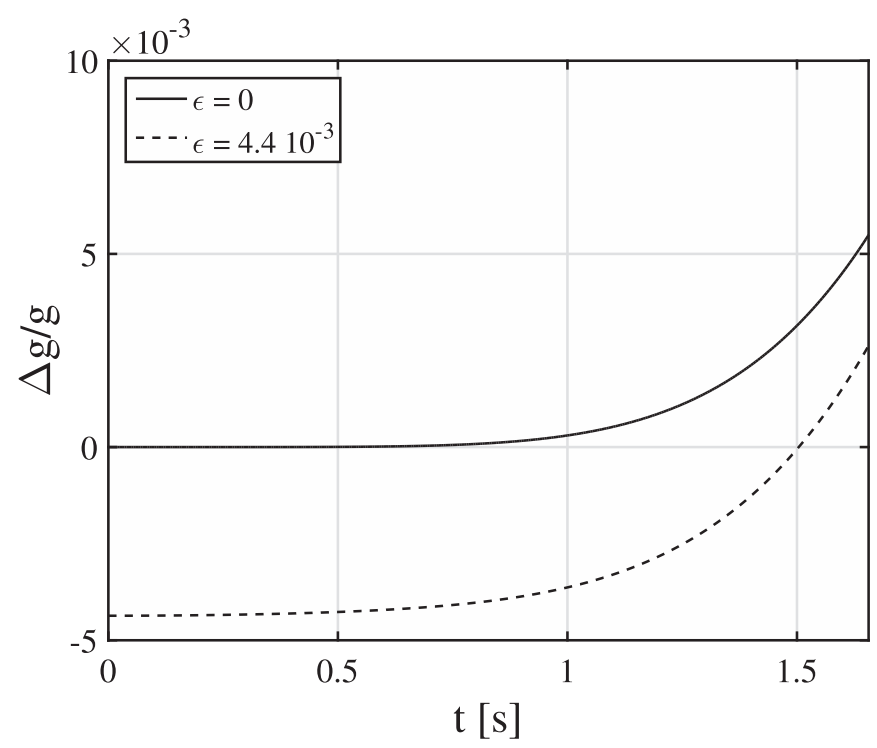

Figure 2. Variation with time $t$, of the microgravity level $\triangle g / g$ at the IDR/UPM drop tower.

$$
F_{\mathrm{p}}=\rho l S_{\mathrm{p}}\left(g-\frac{\rho S C_{\mathrm{D}}}{2 m} v^{2}\right)
$$

According to this argumentation, the acceleration of the platform experiment is

$$
\frac{\mathrm{d} u}{\mathrm{~d} t}=g+\frac{\rho l S_{\mathrm{p}}}{m_{\mathrm{p}}}\left(g-\frac{\rho S C_{\mathrm{D}}}{2 m} v^{2}\right)-\frac{\rho S_{\mathrm{p}} C_{\mathrm{Dp}}}{2 m_{\mathrm{p}}}(u-v)^{2} .
$$

Finally, the microgravity level is given by

$$
\frac{\Delta g}{g}=\frac{1}{g}\left(g-\frac{\mathrm{d} u}{\mathrm{~d} t}\right)=\frac{\rho S_{\mathrm{p}} C_{\mathrm{Dp}}}{2 m_{\mathrm{p}} g}(u-v)^{2}-\epsilon\left(1-\frac{\rho S C_{\mathrm{D}}}{2 m g} v^{2}\right) .
$$

In equation (7), it is observed that the influence of the buoyancy force is proportional to the dimensionless parameter $\epsilon=\rho l S_{\mathrm{p}} / m_{\mathrm{p}}$, which compares the mass of the air displaced by the platform with its mass. The heavier the experimental platform, including the payload, the less important is the effect of the buoyancy. Since the experimental platforms are usually heavy, $\epsilon$ is a small number. Two different cases have been analysed to study the platform movement. In the first one, it is considered that $\epsilon$ is zero, while in the second this parameter has been retained in the equations.

Equations (1), (6) and (7) have been numerically resolved to obtain the microgravity level $\Delta g / g$ and the expected relative height $h_{u v}$ (see figure 1) in the IDR/UPM drop tower. The results are shown in figures 2 and 3 .

To estimate the value of $C_{\mathrm{Dp}}$, the model from White (2010) for the theoretical losses which take place in the sudden expansion of a viscous flow in a circular duct has been used. In this expression the effect of the friction originated in the walls is included 


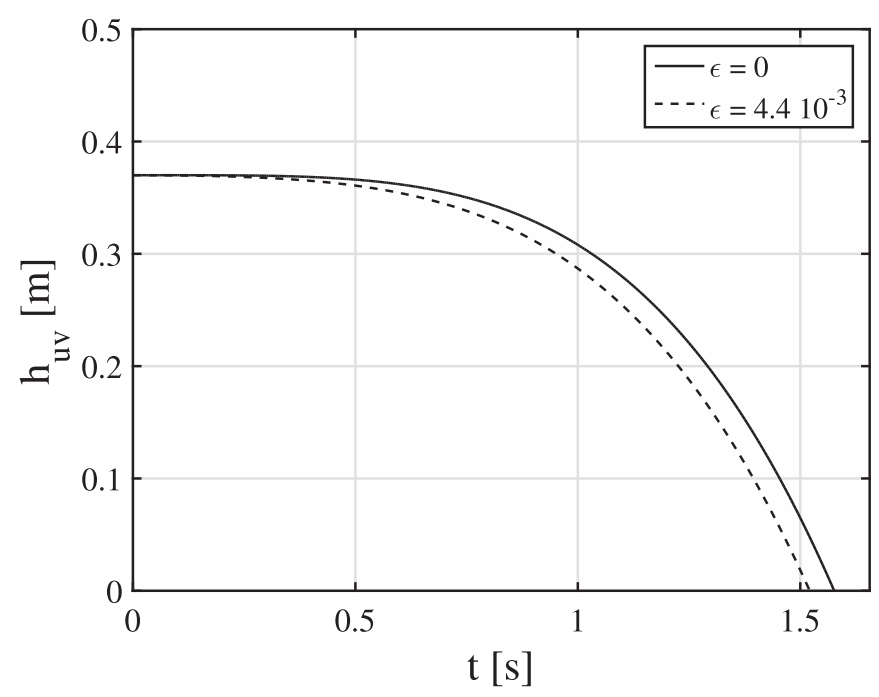

Figure 3. Variation with time $t$, of the relative height $h_{u v}$ at the IDR/UPM drop tower.

Table 1. Typical values at the IDR/UPM drop tower.

\begin{tabular}{lcccccccc}
\hline$m(\mathrm{~kg})$ & $m_{\mathrm{p}}(\mathrm{kg})$ & $S\left(\mathrm{~m}^{2}\right)$ & $S_{\mathrm{p}}\left(\mathrm{m}^{2}\right)$ & $C_{\mathrm{D}}$ & $C_{\mathrm{Dp}}$ & $\rho\left(\mathrm{kg} \mathrm{m}^{-3}\right)$ & $g\left(\mathrm{~m} \mathrm{~s}^{-2}\right)$ & $l(\mathrm{~m})$ \\
3.8 & 5.5 & 0.07 & 0.049 & 0.7 & 9.0 & 1.2 & 9.8 & 0.4 \\
\hline
\end{tabular}

$$
C_{\mathrm{Dp}}=\frac{1+\left(\frac{l}{d_{\mathrm{h}}}\right) f}{\left(\frac{s}{S_{\mathrm{p}}}-1\right)^{2}},
$$

where $d_{\mathrm{h}}$ is the hydraulic diameter, $d_{\mathrm{h}}=2 \sqrt{\left(S-S_{\mathrm{p}}\right) / \pi}$, and $f$ is the friction factor. In general, $f$ depends on the Reynolds number, based on the hydraulic diameter, and the relative roughness. The influence of the relative roughness has been neglected in the analysis. Equation (8) indicates that $C_{\mathrm{Dp}}$ depends on the relative velocity to the air contained in the drag-shield. As first approach and to gain clarity in the study, $C_{\mathrm{Dp}}=9.0$ has been used as mean value. The values of the mean parameters at the IDR/UPM drop tower are shown in table 1.

To gain deeper knowledge and clarity, the problem can be studied in dimensionless parameters, defining $\tau=t / t_{\mathrm{g}}$ as the dimensionless time and $v_{\mathrm{c}}=g t_{\mathrm{g}}$ as the characteristic velocity, where $t_{\mathrm{g}}$ is the drop time that can be calculated by integrating equation (1). Including these definitions in equations (1), (6) and (7) results in

$$
\begin{aligned}
& \frac{\mathrm{d} \tilde{v}}{\mathrm{~d} \tau}=1-\Lambda_{1} \widetilde{v}^{2}, \\
& \frac{\mathrm{d} \tilde{u}}{\mathrm{~d} \tau}=1+\epsilon\left(1-\Lambda_{1} \widetilde{v}^{2}\right)-\Lambda_{2}(\widetilde{u}-\widetilde{v})^{2},
\end{aligned}
$$




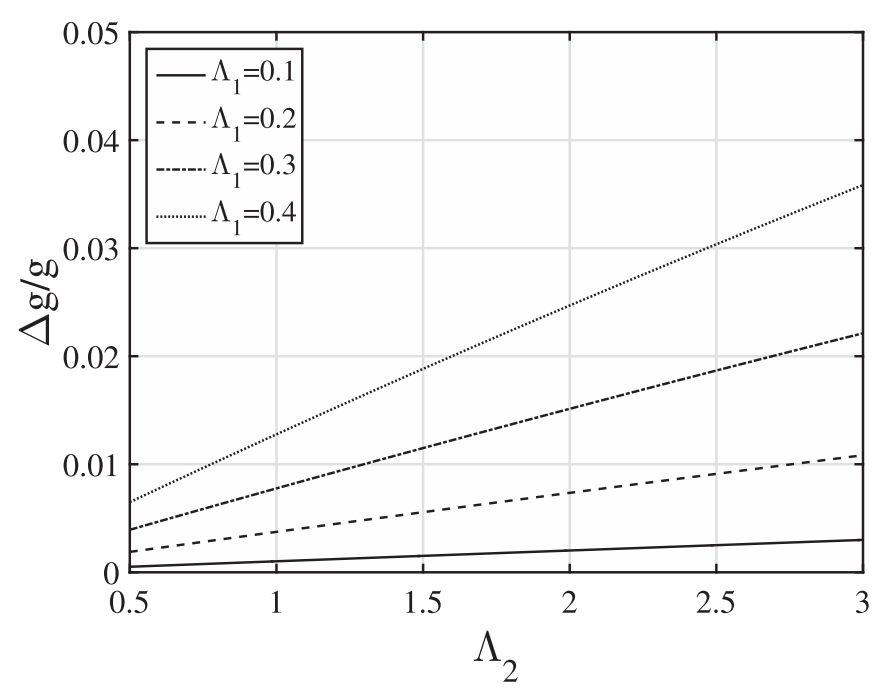

Figure 4. Microgravity level $\Delta g / g$, at the end of the drop for typical values of $\Lambda_{1}$ and $\Lambda_{2}$ when $\epsilon=0$.

Table 2. Estimated values of $\Lambda_{1}$ and $\Lambda_{2}$ in several drop towers with drag-shield operation.

\begin{tabular}{lllll}
\hline$t_{\mathrm{g}}(\mathrm{s})$ & $\Lambda_{1}$ & $\Lambda_{2}$ & Drop tower institution & References \\
\hline 2.1 & 0.3 & 2.5 & INTA & Kaldeich (1995) \\
2.0 & 0.2 & 0.8 & QUT & Steinberg (2008) \\
& & & & Osborne and Welch (2012) \\
1.6 & 0.2 & 1.3 & IDR/UPM & Meseguer et al (2014) \\
\hline
\end{tabular}

$$
\frac{\triangle g}{g}=\Lambda_{2}(\widetilde{u}-\widetilde{v})^{2}-\epsilon\left(1-\Lambda_{1} \widetilde{v}^{2}\right),
$$

where $\tilde{u}=u / g t_{\mathrm{g}}, \tilde{v}=v / g t_{\mathrm{g}}, \Lambda_{1}=\rho S C_{\mathrm{D}} g t_{\mathrm{g}}^{2} / 2 m$ and $\Lambda_{2}=\rho S_{\mathrm{p}} C_{\mathrm{Dp}} g t_{\mathrm{g}}^{2} / 2 m_{\mathrm{p}}$. The above system has been numerically solved for different values of the parameters which can be found in drop towers that use a drag-shield configuration (see table 2).

The parameters $\Lambda_{1}$ and $\Lambda_{2}$ compare the influence of the aerodynamic drag of the bodies with their weight. For lower values of $\Lambda_{1}$ and $\Lambda_{2}$ the aerodynamic drag has less influence on the movement of the bodies. These parameters also indicate that the drag-shield aerodynamic design becomes more restrictive as the height of the drop tower increases, since $\Lambda_{1}$ and $\Lambda_{2}$ are proportional to the square of the drop time.

If $\epsilon$ is neglected, the residual acceleration is zero initially, and increases due to the aerodynamic force until the drag-shield reaches the deceleration system. The maximum of the residual acceleration, which is reached at the end, it is shown in figure 4 for typical values of the parameters $\Lambda_{1}$ and $\Lambda_{2}$. This figure shows the importance of aerodynamic design to improve low gravity conditions, when $\epsilon$ is zero.

If $\epsilon$ is retained in the analysis, the buoyancy effect considerably affects the movement. The residual acceleration is equal to $-\epsilon$ at the release instant and then increases due to the 

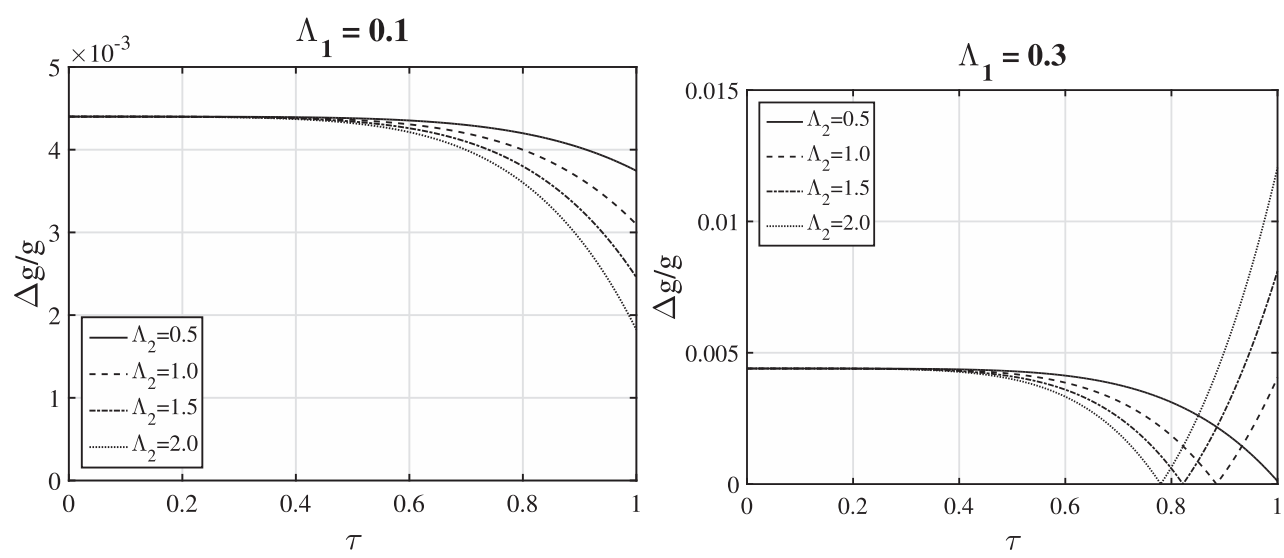

Figure 5. Variation of absolute value of microgravity level with dimensionless time $\tau$, for different values of $\Lambda_{2}$ and $\Lambda_{1}$, with $\epsilon$ equal to $4.4 \cdot 10^{-3}$.
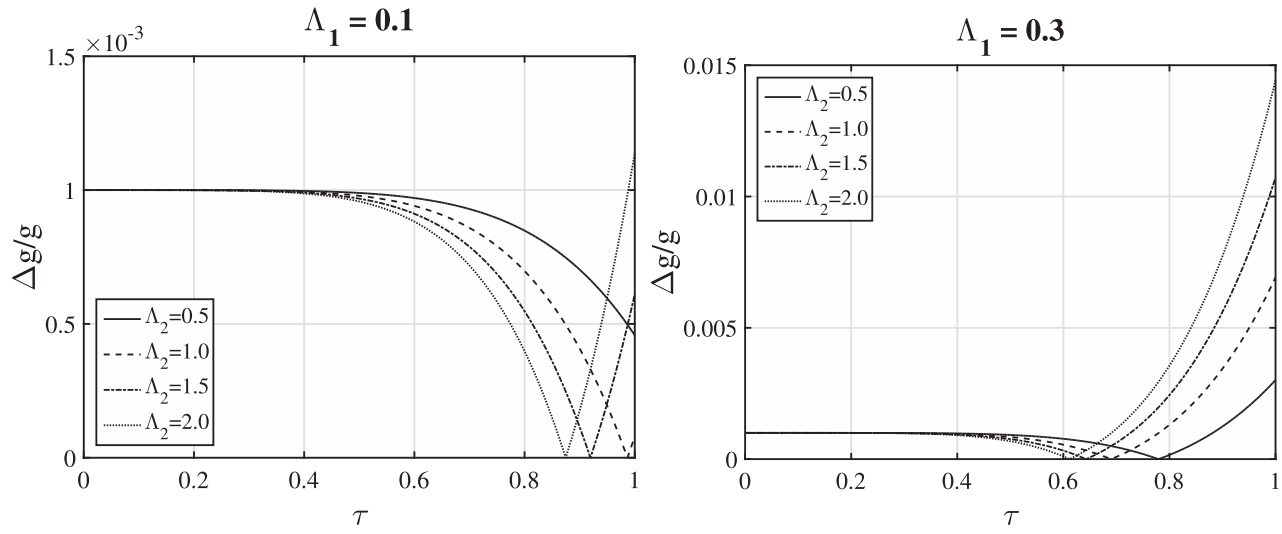

Figure 6. Variation of absolute value of microgravity level with dimensionless time $\tau$, for different values of $\Lambda_{2}$ and $\Lambda_{1}$, with $\epsilon$ equal to $10^{-3}$.

aerodynamic drag. Hence, it is possible that the residual acceleration becomes zero during the drop.

Since the variation of the residual acceleration depends mainly on the aerodynamic drag, if the values of the parameters $\Lambda_{1}$ and $\Lambda_{2}$ are small, the residual acceleration will not vary much from $-\epsilon$. This implies that, if $\epsilon$ is not small, it is better that $\Lambda_{1}$ and $\Lambda_{2}$ are not small either, this way the aerodynamic drag keeps the residual acceleration close to zero over a longer time period. Otherwise, if $\epsilon$ is a very small number, the best low gravity conditions are achieved with small values of $\Lambda_{1}$ and $\Lambda_{2}$. This behaviour can be appreciated in figures 5 and 6 .

\section{Experiment validation}

The experiment platform at the IDR/UPM drop tower is enclosed within the drag-shield, as shown in figure 1. The drag-shield is composed of a cylindrical shroud made of aluminium, a truncated cone also made out of aluminium and a steel spike used to decelerate the whole 


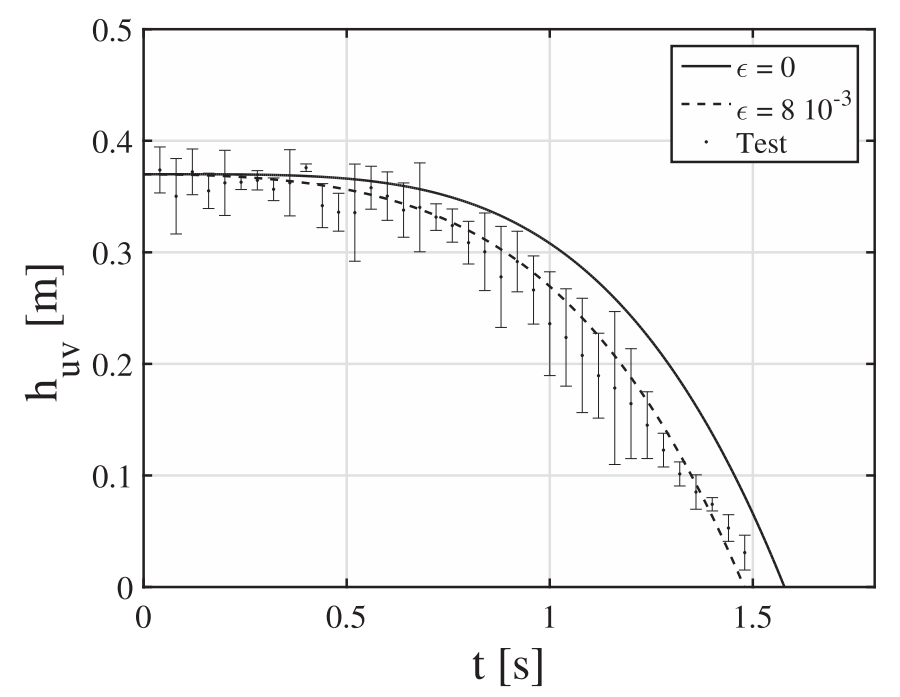

Figure 7. Variation of the relative height $h_{u v}$ with time $t$, at the IDR/UPM drop tower. The experimental data are shown as a dot (mean value) and error bar (standard deviation).

payload. The cone is filled with foam to attenuate as much as possible the deceleration loads on the experiment platform. The experimental platform is a structure composed of four metallic vertical bars that support three equally spaced circular trays. The effective height of the IDR/UPM drop tower for free falling is $13 \mathrm{~m}$, providing about $1.6 \mathrm{~s}$ of drop time. The payload can be elevated at any height between 0 and $13 \mathrm{~m}$, so that the drop time is controlled through the capsule releasing height. The drop package measures $250 \mathrm{~mm}$ in diameter and $400 \mathrm{~mm}$ in height, and is able to support an experimental apparatus weighing a maximum of $15 \mathrm{~kg}$. The payload elevation system is a winch equipped with a dc electric motor provided with the necessary control devices to move the release mechanism up and down, which consists of a lock driven by a solenoid, mounted on a horizontal bar anchored to the hoist cable. When the release mechanism is unlocked, the drop package can free fall down to the base of the tower, where it is decelerated by a large spike that penetrates a dry sand reservoir, the setup is described in more detail in Meseguer et al (2014).

To validate the theoretical models different experiments can be performed. One option could be to measure the acceleration of the platform during the drop, but this would require the use of an expensive accelerometer with high sensitivity to measure the residual acceleration and at the same time support the shock impact, which implies expensive devices. Another possibility is to register the relative height $h_{u v}$ between the platform and the drag shield since the models predict dissimilar evolution during the drop.

To measure the distance between the platform and the drag-shield, an infra-red distance sensor Sharp GP2Y0A21YK0F was used. This sensor can measure distances between 10 and $80 \mathrm{~cm}$ at a sampling frequency of $25 \mathrm{~Hz}$. Data acquisition was performed using an Arduino microcontroller, to keep the analog output provided by the distance sensor on an SD card, and a RaspberryPi controlled from the drop tower control room by a Wi-Fi connection, allowing real-time data and duplication of the measurement.

The platform was made lighter to increase the parameter $\epsilon$ making it easier to appreciate the difference between the models. The mass of the experiment platform, $m_{\mathrm{p}}$, was $3 \mathrm{~kg}$, as a 


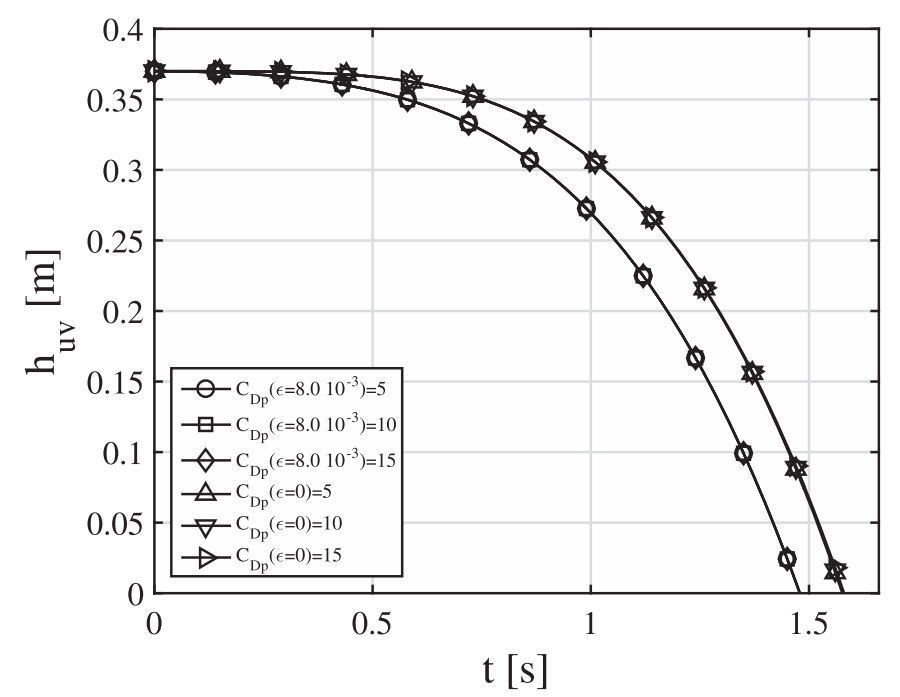

Figure 8. Variation of the relative height $h_{u v}$ with time $t$, at the IDR/UPM drop tower for different values of $C_{\mathrm{Dp}}$.

result $\epsilon$ was $8 \cdot 10^{-3}$. The result of the experiment is shown in figure 7 along with the predictions of the mathematical models.

According to this test, it can be concluded that the model that considers the effect of the buoyancy force is closer to the real movement than the model without buoyancy effect.

At this point, it is important to highlight that the parameter $C_{\mathrm{Dp}}$, which has not been calculated accurately, barely affects the evolution of $h_{u v}$ as is shown in figure 8 .

Although more tests should be performed to study the movement, the experiment in the IDR/UPM drop tower suggests that the buoyancy intervenes in the movement of the experimental platform, and therefore, its effect should be included in the analysis of the microgravity level at drop towers which employ a drag-shield configuration.

The completed model suggests that a convenient strategy to achieve a high microgravity level consists of reducing $\epsilon$, along with $\Lambda_{1}$ and $\Lambda_{2}$. One possible method is to evacuate the air contained within the drag-shield since $\epsilon$ and $\Lambda_{2}$ are proportional to the air density enclosed in the drag-shield. Reducing the inside air density by a factor of 100 , the residual acceleration would be hundred times smaller. However, the application of this method requires redesigning the drag-shield to ensure sealing as well as incorporating a system to evacuate air from the drag-shield. In addition, the difference in pressure makes the structure sensitive to buckling in the deceleration period, so the drag-shield should be reinforced.

Another possibility to reduce the residual acceleration is the dual drag-shield. This strategy consists of introducing a second drag-shield between the platform and the outside drag-shield in order that the aerodynamic drag which affects the platform would be even smaller. The equations that describe the movement are similar to those shown above, considering that those on the inside drag-shield also act the aerodynamic drag and the buoyancy. By applying this method, the residual acceleration is drastically reduced if the buoyancy effect is not included since the aerodynamic drag is the only force that acts on the platform. However, if buoyancy affects the movement, the microgravity level is not improved because the platform movement is dominated by the parameter $\epsilon$. These results are presented in figure 9. 

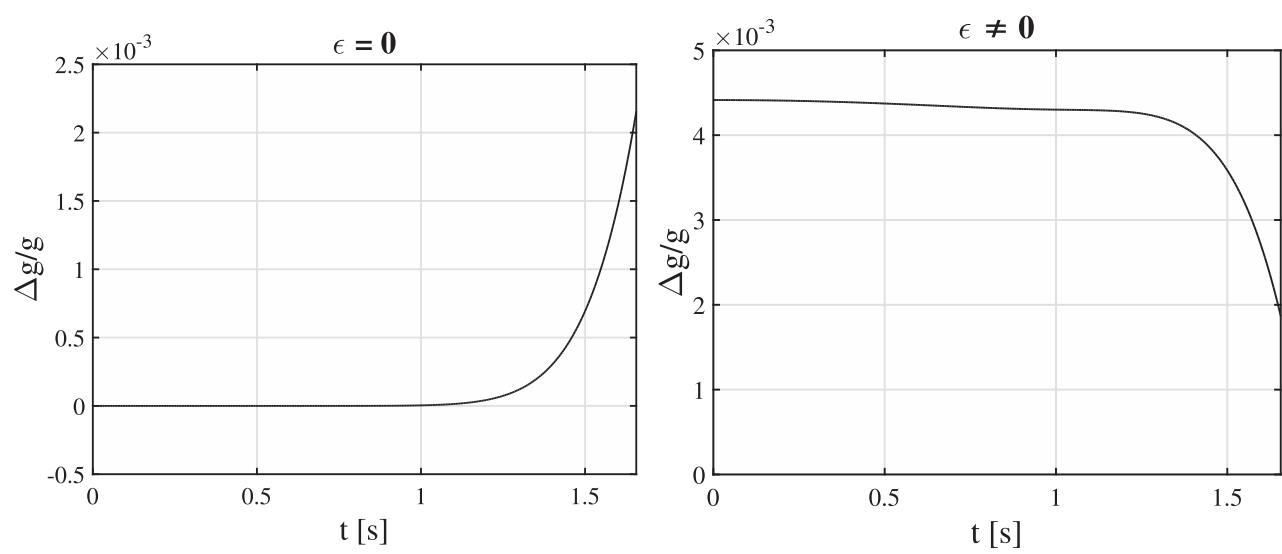

Figure 9. Variation with time $t$, of absolute value of microgravity level $\triangle g / g$ at the IDR/UPM drop tower for the dual drag-shield.

\section{Conclusions}

A theoretical approach of the experiment platform movement at a drop tower with drag-shield configuration has been validated experimentally. The main parameters of the problem of microgravity optimisation in a drop tower with drag-shield configuration have been targeted and the importance of considering buoyancy forces to evaluate the residual microgravity highlighted. Therefore, the conducted study can be used to analyse the efficiency of possible enhancements of drop towers with drag-shield configuration. The dual drag-shield configuration has been analysed and discarded due to the buoyancy effect.

An alternative configuration, based on using a vacuum between the platform and the drag-shield is proposed. The authors believe that future designs with this configuration should be developed as the expected residual acceleration would be very low and could be used in higher drop towers. Drop towers higher than $30 \mathrm{~m}$ usually have the vacuum in the whole tower to reach the microgravity environment (Lekan 1989, Mori et al 1993, Von Kampen et al 2006), which supposes a high operative cost that would be saved with the proposed configuration.

\section{Acknowledgments}

The authors want to thanks Professor J Meseguer for his contribution to this work, without his support this experiments could not be performed. He passed away without being able to see the results of this work. We also want to thank Professor A Sanz-Andres for his help with the models and everyday support during the process.

\section{References}

Kaldeich B 1995 Facilities for Microgravity Investigations in Physical Sciences Supported by ESA (ESA $S P$ vol 1116) (Noordwijk: ESA Publications Division)

Kufner E et al 2011 ESA's drop tower utilisation activities 2000 to 2011 Microgravity Sci. Technol. 23 409-25

Lekan J 1989 Microgravity research in NASA ground-based facilities 27th Aerospace Sciences Meeting (Reno, NV, 9-12 January) (American Institute of Aeronautics and Astronautics) AIAA-89-0236 
Li Z, Liu Q and Liu R 2011 Investigation of gas-liquid interface behavior on propellant reorientation in microgravity environment J. Jpn. Soc. Microgravity Appl. 28 S120-5

Malméjac Y, Bewersdorff A, Da Riva de la Cavada I and Napolitano L G 1981 Challenges \& prospectives of microgravity research in space Technical report (European Space Agency) (http:// oa.upm.es/13868)

Meseguer J, Sanz-Andrés Á, Pérez-Grande I, Pindado S, Franchini S and Alonso G 2014 Surface tension and microgravity Eur. J. Phys. 35055010

Mori T, Goto K, Ohashi R and Sawaoka A B 1993 Capabilities and recent activities of Japan Microgravity Center (JAMIC) Microgravity Sci. Technol. 5 238-42

Osborne B and Welch C 2012 Short duration reduced gravity drop tower design and development JBIS -J. Br. Interplanet. Soc. 65 71-6

Reibaldi G, Manieri P, Mundorf H, Nasca R and König H 2004 The European multi-user facilities for the Columbus Laboratory ESA Bull. 102 107-20

Ruyters U and Friederich G 2006 From the breme drop tower to the International Space Station ISSways to weightlessness in the German space life sciences program Signal Transduction 6397405

Steinberg T 2008 Reduced gravity testing and research capabilities at new 2.0 second drop tower J. Achiev. Mater. Manuf. Eng. 31 822-6

Thomas V A, Prasad N S and Ananda Mohan Reddy C 2000 Microgravity research platforms-a study Curr. Sci. 79 336-40

Urban B D, Kroenlein K, Kazakov A, Dryer F L, Yozgatligil A, Choi M Y, Manzello S L, Lee K O and Dobashi R 2004 Sooting behavior of ethanol droplet combustion at elevated pressures under microgravity conditions Microgravity-Sci. Technol. 15 12-18

Von Kampen P, Kaczmarczik U and Rath H J 2006 The new drop tower catapult system Acta Astronaut. $59278-83$

White F M 2010 Fluid Mechanics (McGraw-Hill Series in Mechanical Engineering) 7th edn (Columbus, OH: McGraw-Hill Education) 